\title{
Is combination antithrombotic therapy safe for patients with atrial fibrillation?
}

Patients with atrial fibrillation (AF) often receive anticoagulant and antiplatelet therapy to reduce thrombotic events, including stroke. However, few studies have examined the risks associated with combination therapy. The risk of bleeding following treatment with warfarin, aspirin, clopidogrel, and combinations of these drugs was assessed in an observational study of 118,606 patients with AF discharged from hospitals in Denmark between 1997 and 2006. "We believe that it is imperative that better estimates of safety are provided in terms of the risk of bleeding complications," says investigator Morten Hansen.

During follow-up (mean 3.3 years), the combined end point (nonfatal and fatal bleeding) occurred in $11.4 \%$ of patients. Patients receiving warfarin and clopidogrel, or triple therapy, had a more-than-threefold higher risk of bleeding than patients treated solely with warfarin. In addition, combination therapy had no beneficial effect in terms of preventing ischemic stroke. These findings highlight that dual and triple antithrombotic therapies have a worse safety profile than previously recognized. "Combination therapy should be carefully considered and should only be given for a short time when treatments are mandatory," warns Hansen.

Following on from this work, Hansen and colleagues are aiming to address two important questions: what is the risk of thromboembolism after discontinuing oral anticoagulant therapy in patients with $\mathrm{AF}$, and when does bleeding occur after initiation of triple therapy with aspirin, clopidogrel and warfarin?

Helene Myrvang

Original article Hansen, M. L. et al. Risk of bleeding with single, dual, or triple therapy with warfarin, aspirin, and clopidogrel in patients with atrial fibrillation. Arch. Intern. Med. 170, 1433-1441 (2010) 